\title{
Résultat et performance financière en normes IFRS : Quel est le contenu informatif du comprehensive income?
}

\author{
Olivier RAMOND, Laurent BATSCH et Jean-François CASTA \\ Centre de recherches en Gestion (DRM-CEREG UMR 7088) - Université Paris-Dauphine
}

Cahier de recherche $n^{\circ}$ 2007-04

Résumé : L'IASB et le FASB ont formé en 2003 un groupe de travail commun en vue de faire avancer le projet de création de normes de reporting de la performance financière. Intitulé à l'origine "Performance Reporting » et se référant à une conception étendue du résultat — le comprehensive income - , ce projet pose avec acuité la question de l'utilité du concept traditionnel de « résultat net » dans le référentiel international. Le présent article analyse la légitimité de l'introduction du comprehensive income dans le cadre du projet «Performance Reporting » au regard des critères de qualité de l'information édictés par l'IASB. A partir d'un échantillon d'entreprises françaises et britanniques cotées, observées sur la période préIFRS (1992-2004) et post-IFRS (2005), nous examinons la value-relevance et l'utilité informationnelle de trois mesures de performance : le résultat net, le résultat opérationnel et le comprehensive income. Nous mettons empiriquement en évidence: (1) leur association respective avec les rendements boursiers, (2) la dominance, en variable agrégée, du résultat net sur le comprehensive income, (3) l'information additionnelle, par rapport au résultat net, apportée par les other comprehensive income.

Mots-clés :

IFRS, Résultat global, comprehensive income, dirty surplus, résultat net, reporting de la performance, degré de pertinence, projet Performance Reporting de l’IASB-FASB.

\section{Summary Accounting Income Measures and Performance Reporting Under IFRS: What is the Information Content of Comprehensive Income?}

Abstract: In 2003, the IASB and the US FASB formed a Joint International Group (JIG) whose objective was to carry out a project originally untitled "Performance Reporting" establishing new international financial reporting standards for performance reporting. By envisaging the use of a comprehensive income (CI) item similar to the one reported under US GAAPs, the current debate surrounding this project is slowly deviating from its technical premises to the more fundamental issue related to accounting income usefulness. In this article, we investigate whether the focus on a CI item is legitimate regarding the IASB informational criteria. Using a dataset made of French and UK listed companies over the pre(1993-2004) and post-(2005) IFRS compliance period, we examine the value-relevance and usefulness of three summary income measures (i.e. operating, net and comprehensive income) and bring evidence that (1) they are all associated with share return, (2) net income dominates $\mathrm{CI}$ at an aggregate level and (3) OCI provide incremental information beyond net income.

Keywords :

IFRS, Comprehensive income, Dirty surplus flow, Summary income measures, Valuerelevance, IASB/FASB "Performance Reporting” project. 


\section{Résultat et performance financière en normes IFRS : Quel est le contenu informatif du comprehensive income?}

\section{Introduction}

Le projet de «Performance reporting » de l'IASB, amorcé en 2001, puis relancé en 2004, a pour objet d'édicter une norme portant sur la communication des performances financières et comptables des entreprises soumises aux IFRS. En se référant à une conception étendue du résultat — le comprehensive income (CI) —, ce projet pose avec acuité la question de l'utilité du concept traditionnel de « résultat net » dans le référentiel international. Le comprehensive income diffère fondamentalement du concept classique de résultat net, car il inclut les variations de capitaux propres (autres que les opérations en relation avec les actionnaires), comme les plus ou moins values en capital, réalisées ou non, provenant de la réévaluation de certains actifs et de certains passifs.

Le comprehensive income procède d'une re-construction de la notion de « Résultat » et de celle de performance qui, au-delà d'une apparence technique, revêt une réalité conceptuelle. L'apparence technique correspond à une approche en termes de tableau de passage du résultat net au comprehensive income, s'intercalant entre le compte de résultat et le tableau de variation des capitaux propres. L’approche en termes de comprehensive income privilégie les états financiers orientés vers le bilan, au détriment des états financiers plus centrés sur le compte de résultat. Le fondement conceptuel sous-jacent porte en lui une évolution plus radicale : le « Résultat » pertinent serait celui qui mesure la variation de la valeur du capital, c’est-à-dire, en définitive, l’enrichissement des actionnaires. Le « Résultat » ne serait plus une différence entre des produits et des charges enregistrés en coût historique sur une période, mais la différence entre deux «stocks » de capital mesurés en valeurs de marché ou d'utilité. En effet, pour tout investisseur, le revenu a deux origines : le flux (dividendes, coupons...) et la plus-value. La conception élargie du résultat ne fait qu’intégrer l’approche financière de la 
performance dans le cadre de la comptabilité. En ce sens, ce mouvement s’inscrit dans la logique de financiarisation du modèle comptable engagée par l'IASB avec l'utilisation progressive de la convention d'évaluation en juste valeur (Casta et Colasse, 2001).

Cet article se propose d'analyser la légitimité de l’introduction du comprehensive income dans le cadre normatif international au regard des critères informatifs édictés par l’IASB. Afin de contribuer à ce débat, nous utiliserons une méthodologie d'étude de value-relevance des composants du résultat dans la lignée des travaux fondateurs (Abarbanell et Bushee (1988) ; Amir, Harris et Veuti (1993)). À cette fin, nous nous proposons de tester empiriquement, sur la période pré-IFRS (1993-2004) et post-IFRS (2005), la value-relevance de différentes mesures du résultat (i.e. résultat opérationnel, résultat net, comprehensive income) et de leurs composants. Afin de procéder à cette étude dans un contexte européen suffisamment différencié au plan réglementaire, sociologique et économique, nous avons retenu les marchés financiers français et britanniques : en effet, la France présente un exemple d’absence de référence au comprehensive income dans ses normes comptables nationales ; inversement, les UK GAAP illustrent une pratique de divulgation du comprehensive income qui remonte à 1992.

Le présent article est structuré de la façon suivante : nous présenterons en premier lieu de l'émergence du concept de comprehensive income au regard du débat institutionnel (section 1) ; nous examinerons les recherches traitant du contenu informatif des différentes mesures de résultat, avant de présenter la méthodologie de test de leur value-relevance et de leur utilité (section 2) ; les données utilisées dans l'étude empirique seront ensuite détaillées (section 3) ; enfin, nous analyserons et nous discuterons les résultats obtenus (section 4). 


\section{L’émergence du comprehensive income dans le débat institutionnel}

\subsection{Reporting de la performance : quel est le besoin présumé des utilisateurs ?}

L’International Accounting Standards Board (IASB) et le Financial Accounting Standards Board (FASB) ont formé en 2003 un groupe de travail commun, dénommé «Joint International Group » (JIG) en vue d’améliorer la communication et la présentation des états financiers. Ce projet, encore à l'étude, originellement nommé «Performance Reporting : Reporting Comprehensive income » a pour objet :

«[...] d'établir des normes sur la présentation de l'information afin d'améliorer l'utilité de cette information et donc de mieux évaluer les performances financières et la situation financière d'une entité » (IASB, Project Overview, 25 octobre 2004).

Ce projet devrait aboutir à la première norme harmonisant le reporting des performances financières en référentiel IFRS. Certains académiques, auxquels se joignent des professionnels (cf. Linsmeier et al., 1997 ; Barker, 2004), jugent ce projet particulièrement opportun en raison de la prolifération des mesures de performance financière utilisées, dans leur communication, par les sociétés cotées. La multiplicité de ces mesures et leur inconsistance conceptuelle s’avéreraient préjudiciables à la qualité du reporting financier et $a$ fortiori à l’efficacité des marchés de capitaux (Lin, 2006).

Bien qu'apparemment limité à un débat d'ordre technique, ce projet est à l'origine d'une importante polémique qui porte sur la mesure de la performance financière d’une entreprise. Dans cette nouvelle perspective, les sociétés soumises aux IFRS seraient tenues de divulguer le comprehensive income, une mesure de «résultat global » d'origine anglo-saxonne fortement controversée. Le comprehensive income (également appelé all-inclusive income ou clean-surplus income) est défini par le Concepts Statement $N^{\circ} 6$ 'Elements of Financial Statements' du FASB (1985), comme “the change in equity of an entity during a period from 
transactions and other events other than those resulting from contributions by and distributions to equity holders in their capacity as equity holders" (SFAC nº, paragraphe 12).

En phase avec la réglementation comptable américaine, l'Exposure Draft 'Presentation of Financial Statements' (i.e. la proposition d'amendement à la norme IAS 1) de l'IASB, publié en mars 2006, insiste particulièrement sur le « Total recognised income and expense ", défini comme "the change in equity of an entity during a period from transactions and other events other than those resulting from contributions by and distributions to equity holders in their capacity as equity holders” (IASB, 2006, BC 17: 78). Cette nouvelle ligne des états financiers renvoie directement au concept américain de comprehensive income et transpose sur la scène comptable européenne le débat sous-jacent.

\subsection{Other Comprehensive Income : état des pratiques}

Le comprehensive income diffère du concept classique de résultat net dans la mesure où il inclut certaines variations de capitaux propres non occasionnées par des transactions avec les détenteurs du capital (i.e. les distributions de dividendes et les opérations sur capital). Il s’agit de gains ou de pertes en capital (plus ou moins values) réalisés ou non, provenant de la réévaluation de certains actifs et de certains passifs. Nommés dirty surplus items par les académiques et other comprehensive income (OCI) par les professionnels, leur traitement contourne, pour des raisons exclusivement normatives, propres à chaque contexte réglementaire, le compte de résultat et impacte directement les capitaux propres d’une entité. Les exemples les plus courants d’OCI sont les écarts sur réévaluation d’actifs, les écarts actuariels, les écarts de change, les écarts de conversion, les plus-values latentes sur les actifs financiers disponibles à la vente, les variations de valeur des instruments dérivés servant de couverture des flux de trésorerie ou certains compléments de provisions pour retraite... Avec 
la généralisation de la comptabilité en juste valeur, la tendance est à un développement de la pratique des OCI (dépréciation de goodwill, réévaluation d'actifs) qui permet d'éviter toute déstabilisation exceptionnelle du résultat net et assure, dans une certaine mesure, sa stabilité au cours du temps.

\subsubsection{Comptabilité de clean surplus vs. comptabilité de dirty surplus ${ }^{1}$}

Le développement de la pratique des OCI, tant au niveau local qu'international, oppose deux conceptions de l'articulation comptable reliant les capitaux propres et le résultat d'une entreprise : la comptabilité de clean surplus et la comptabilité de dirty surplus.

Dans la comptabilité de clean surplus, les variations des capitaux propres autres que les opérations en relation avec les actionnaires (i.e. les distributions de dividendes et les opérations sur le capital) sont intégrées au résultat de l’exercice. A l’opposé, une comptabilité est dite de dirty surplus si certaines variations des fonds propres ne sont pas reflétées dans le compte de résultat. Les référentiels comptables permettent en effet à certains postes comptables, appelés dirty surplus items, de contourner arbitrairement le compte de résultat. Cependant, les différents référentiels comptables n’intègrent pas les mêmes éléments de dirty surplus (voir infra tableau 1).

La figure 1 illustre, de façon synthétique, la relation entre le résultat global (ou comprehensive income) et le résultat net de l'exercice. Les «ajustements comptables accumulés » et les « autres changements de capitaux propres non attribuables aux actionnaires » représentent alors les dirty surplus.

Figure 1. Relations entre le résultat net et le résultat global

\footnotetext{
Résultat net

+ / - ajustements comptables accumulés

$+/$ - autres changements de situation nette non attribuables aux actionnaires

= Résultat global (ou comprehensive income)
} 
Selon cette approche, la variation de situation nette comptable (figure 2) peut ainsi être directement déduite du montant du résultat global associé aux autres variations financières des capitaux propres (distribution de dividendes et changements de capital).

\section{Figure 2. Relations entre la situation nette comptable et le résultat global}

\begin{tabular}{l} 
Capitaux propres au 1/01/N \\
+ Résultat global (ou Comprehensive Income) \\
+ Changements de capital (émission de titres et rachat d'actions propres) \\
- Distribution de dividendes \\
\hline = Capitaux propres au 31/12/N \\
\hline
\end{tabular}

Les partisans de la comptabilité en dirty surplus, dont le Professeur Francis Black ${ }^{2}$ était le chef de file au début des années 1980, soutiennent que seule une métrique de résultat fondée sur le concept de «bénéfice durable » (sustainable earnings) — i.e. épurée des éléments transitoires et des flux hors exploitation — peut avoir une utilité informationnelle au regard du processus d'évaluation des actions.

Avec des objectifs similaires, les partisans de la comptabilité en clean surplus — dont font désormais partie la plupart des instances comptables anglo-saxonnes - , souhaitent promouvoir l'efficience des marchés en orientant le système comptable vers une perspective plus financière, directement propice à l'évaluation des actifs d'une entreprise par un agent économique extérieur.

\subsubsection{UK GAAPs : une adoption précoce du comprehensive income}

La première impulsion a été donnée dans ce sens par l’Accounting Standards Board (ASB) britannique. En effet, le concept de comprehensive income est apparu, en 1992, avec la publication de la norme FRS 3 «Reporting Financial Performance » relative à la communication de la performance financière des sociétés cotées. Cette norme pose le problème de la mesure globale de la performance : elle introduit un nouveau compte de 
résultat appelé «Statement of Total Recognized Gains and Losses ». Cette novation met l'accent sur les composants de la performance financière et crée un état intermédiaire entre le compte de résultat au coût historique et le bilan. La norme FRS 3 prévoit que le compte de profits et pertes (profit \& loss account) doit présenter les composants suivants de la performance financière :

- The results of continuing operations,

- The results of discontinued operations,

- Gains and Losses,

- Extraordinary items.

Cette reformulation par IFRS 3 du concept de performance financière a deux objectifs : d'une part, permettre aux utilisateurs des états financiers de mesurer la performance globale de l'entreprise et, d'autre part, fournir l'information nécessaire à l'évaluation des résultats et des cash flows futurs en mettant en évidence l'ensemble des transactions enregistrées au cours de l’exercice, y compris celles ayant contourné le compte de résultat.

\subsubsection{US GAAPs : une divulgation flexible du comprehensive income}

Dans la lignée du FRS 3 précédemment adopté au Royaume-Uni, la norme SFAS 130 intitulée Reporting comprehensive income introduit, en 1997, un nouvel état de synthèse, appelé Statement of other comprehensive income. Le comprehensive income mesure la variation des capitaux propres d'une entreprise, pendant une période, résultant des transactions et d'autres événements, excepté celles qui résulteraient des relations avec les actionnaires. Ce concept de revenu global inclut tous les revenus, les dépenses, les gains et les pertes identifiés pendant la période, le revenu net devenant un composant du revenu global. Cette norme ne prescrit aucun format spécifique d’état financier. Elle exige des entreprises une présentation 
permettant le passage du résultat net (net income) au comprehensive income, permettant ainsi une mesure de la variation périodique des capitaux propres, c'est-à-dire de la richesse des actionnaires. En normes américaines, les OCI comprennent : les gains et les pertes potentiels sur des titres de placement (unrealised holding gains and losses on investments in securities classified as availables for sale), les ajustements potentiels sur des coûts de pension de retraite (unrealised pension cost adjustments), les écarts sur opérations de change (foreign currency translation adjustments).

SFAS 130 propose de présenter les OCI sous trois formats différents : (1) dans le même état financier que celui du revenu net, (2) dans un état financier spécifique ou (3) dans le tableau de variation des capitaux propres.

\subsubsection{La pratique des OCI en référentiel IAS / IFRS}

En référentiel IAS / IFRS, de nombreuses normes se réfèrent à l'utilisation implicite ou explicite d'OCI, nommés Other recognized income and expense (ORIE) par l’IASB. Le projet de refonte de la norme IAS 1 "Présentation des états financiers " ${ }^{3}$ énonce de manière exhaustive les ORIE dans son paragraphe 7 :

« The components of other recognised income and expense are:

(a) changes in revaluation surplus (see IAS 16 Property, Plant and Equipment and IAS 38 Intangible Assets);

(b) gains and losses arising from translating the financial statements of a foreign operation (see IAS 21 The Effects of Changes in Foreign Exchange Rates);

(c) gains and losses on remeasuring available-for-sale financial assets (see IAS 39 Financial Instruments: Recognition and Measurement);

(d) the effective portion of gains and losses on hedging instruments in a cash flow hedge (see IAS 39); and 
(e) actuarial gains and losses on defined benefit plans recognised in accordance with paragraph 93A of IAS 19 Employee Benefits. »

Cette volonté de divulguer, de manière obligatoire, les ORIE en référentiel international fait parti intégrante de la politique de convergence des normes US GAAPs avec les normes IFRS lancée conjointement par l'IASB et son homologue américain. Ces deux instances ne semblent pas prêtes à abandonner l'idée de l'introduction du comprehensive income dans les états financiers des entreprises communiquant en IFRS. Les responsables du projet de convergence ont déclaré, lors de la réunion FASB / IASB du 28 avril 2004, qu’une « période pédagogique » serait nécessaire afin de dépasser la controverse relative à l'utilisation de la notion de comprehensive income, notamment en raison de la non-utilisation de ce concept en dehors des environnements réglementaires anglo-saxons. Sir David Tweedie, président de l’IASB, avait ajouté que la seule image fidèle véritablement représentative de la performance d'une entreprise, publiant ses opérations en normes IFRS, ne pouvait être fondée que sur le résultat global, i.e. le comprehensive income.

\subsubsection{French GAAPs : une utilisation parcimonieuse des OCI}

Comme la plupart des référentiels latins (e.g. Spanish et Italian GAAPs), les normes françaises appuyées sur le règlement CRC n99-02 ${ }^{4}$ permettent une utilisation limitée des OCI. Ces derniers incluent de manière exhaustive :

1) Les gains et pertes de change résultant de l'utilisation de la méthode du taux de clôture (art. 320) ;

2) Les écarts d'acquisition et d'évaluation relatifs à un regroupement d'entreprises en cas de recours à la « méthode dérogatoire » (pooling of interests à la française) (art. 215) ; 
3) Le changement de méthodes comptables (art. 423 et § 2.1.2. de l’avis du CNC n97-06 relatifs aux changements de méthode comptable, changements d'évaluation, changements d'options fiscales et corrections d'erreur) ;

4) Les réévaluations d’actif autorisées par le législateur (art. 312).

\subsubsection{Synthèse des pratiques d'OCI en French, UK, US GAAPs et normes IFRS}

Résumant l'état des pratiques en matière d’OCI, le tableau 1 présente les différents dirty surplus items autorisés en normes américaines, britanniques, françaises et internationales :

\section{[INSERER LE TABLEAU 1]}

\subsection{De la nécessité de divulguer le comprehensive income : une vision controversée}

Selon les promoteurs du comprehensive income, la comptabilité traditionnelle, percluse d’OCI, montre deux faiblesses majeures. En premier lieu, l'utilisation des OCI engendrerait des biais dans la valorisation des performances par les investisseurs (Barker, 2004) et aggraverait les problèmes d'agence en facilitant les manipulations de résultats par les entreprises (Robinson, 1991 ; CFA Institute, 2005), contribuant ainsi à la baisse de la pertinence des résultats comptables (Cope, Johnson et Reither, 1996). En second lieu, la présence d'éléments de dirty surplus ne repose sur aucun fondement économique ni théorique ; elle résulte d’activités de lobbying ad hoc développées auprès des organismes de normalisation comptable. Dès lors, les divergences culturelles et institutionnelles entre les systèmes comptables nationaux sont sources de pratiques diverses en matière de dirty surplus, réduisant ainsi la comparabilité inter-entreprises des états financiers et donc, pour les investisseurs, celle des performances financières (Linsmeier et al., 1997 ; CFA Institute, 2005). A cet égard, le Chartered Financial Analysts (CFA) Institute, anciennement 
Association for Investment Management and Research (AIMR), un des plus influents groupes internationaux d'utilisateurs d'états financiers, déclare dans son rapport de 1993 intitulé 'Financial Reporting in the 1990s and Beyond', " Nous avons de profondes craintes quant au nombre croissant de variations de valeur qui n’apparaissent pas dans le compte de résultat. Pourtant, certains éléments peuvent être interprétés différemment. C'est la raison pour laquelle le comprehensive income doit être communiqué puisqu'il permet de voir et d'évaluer séparément des éléments de natures différentes » (AIMR, 1993, p. 63). Dernièrement, le CFA Institute a réitéré son appel en soutenant que «la divulgation d'informations doit être opportune, précise, compréhensible et exhaustive afin que l'évaluation [des ressources économiques d'une entreprise et [...] des performances d'une entité] soit utile. Les états financiers doivent enregistrer, au fur et à mesure, tout événement ou transaction qui affectent la valeur de l'actif net d'une entreprise et, donc, la richesse des actionnaires » (CFA Institute, 2005, p.10).

De leur côté, les défenseurs de la comptabilité en dirty surplus ont constamment justifié cette pratique par le fait qu'elle aide à produire une mesure de la performance plus fine, centrée sur les « opérations normales» de l'entreprise et permettant ainsi de faciliter le travail de valorisation (Kiger et Williams, 1977 ; Black, 1993 ; Brief et Peasnell, 1996). De manière identique, Black (1980 ; 1993) affirme que les utilisateurs des états financiers — qui incluent aussi bien les analystes que les actionnaires, les créanciers, les managers, les administrations fiscales et même les économistes — préfèrent une donnée comptable — earnings figure — qui mesure la valeur et non la création de valeur.

Dès lors, l'un des principaux problèmes soulevés par le projet de l'IASB est de savoir si le format du reporting de la performance financière induit une différence significative sur les marchés de capitaux. Cette question revêt une importance particulière pour les petits investisseurs qui, contrairement aux institutionnels, ne peuvent filtrer les informations sans 
supporter des coûts élevés de retraitement. Les auteurs s’accordent à dire que le reporting de la performance financière des entreprises cotées est essentiel : il affecterait la répartition des investissements internationaux (Ball, 1995) par le jeu de divers mécanismes économiques (Zeff, 1978), comme l'impact sur l'asymétrie d'information entre les managers et les investisseurs (Leuz et Verrecchia, 2000 ; Bushman et Smith, 2001). Peu de preuves empiriques ayant été apportées sur les conséquences économiques du reporting financier, les Boards des organismes de normalisation doivent être incités à la prudence lors de l'élaboration d'une nouvelle norme de communication de la performance financière (Barker, 2003 ; 2004).

\section{Cadre d'analyse et méthodologie de recherche}

\subsection{Revue de littérature}

\subsubsection{De la pertinence du résultat net}

Plusieurs travaux abondent dans le sens du constat d'une dégradation de la qualité prédictive de l'information comptable. Francis et Schipper (1999) confirment, sur une très longue période (1952-1994), la baisse de la pertinence de l’information sur les « résultats » et l’amélioration de la pertinence du bilan. Toutefois, l’idée du déclin de la pertinence des chiffres comptables est contestée par plusieurs travaux. Ely et Waymire (1996) testent la force de la relation entre les résultats comptables et les returns boursiers aux Etats-Unis au cours de trois sous-périodes faisant suite à des changements de normes. Ils concluent à l'absence de lien significatif, mais remarquent que l'adoption des normes FASB (1973) a plutôt amélioré la pertinence des chiffres comptables. Collins, Maydew et Weiss (1997) sur la période 19531993 aboutissent à la même conclusion : la pertinence globale des résultats et des valeurs du bilan comptable n’aurait pas diminué. 


\subsubsection{Du contenu informatif du comprehensive income}

L’un des sujets de la controverse porte sur la nécessité de créer un nouvel état de synthèse 5 . Les éléments qui compose le CI sont-ils ou non reliés à la performance ? En cas de réponse positive, ils feraient partie intégrante du résultat et l'élaboration d'un nouvel état de CI s’imposerait. Dans le cas inverse, il serait logique de les enregistrer en dehors du résultat, c’es-à-dire en variation des capitaux propres.

Dans le champ académique, certains protagonistes soutenaient que le tableau de variation des capitaux propres suffisait à intégrer les éléments exceptionnels du résultat. Par exemple, Hirst et Hopkins (1998) soulignent que la bonne connaissance du CI a un effet déterminant sur la qualité des anticipations des analystes. A partir d'un test de comportement sur des analystes buy-side, ils montrent que la présence du CI améliore la qualité de leurs prévisions, davantage que le seul tableau de variation des capitaux propres. La démonstration met en évidence l'intérêt de distinguer les variations des capitaux propres selon leur origine: celles qui relèvent de transactions avec les actionnaires et les autres qui devraient alors isolées dans un document spécifique.

Inversement, Dhaliwal et al. (1999) établissent que les rentabilités observées ne sont pas expliquées par l’un des trois éléments supplémentaires qui viennent s’ajouter au résultat net pour former le CI tout au moins, dans la période pré-SFAS 130, pour les entreprises industrielles. De leur côté, Biddle et Choi (2006) observent que le CI (au sens du FAS 130) domine en termes de degré de pertinence le résultat net. Ils défendent donc un point de vue conciliateur et rappellent que « la norme SFAS 130 recommande la production simultanée de deux définitions du résultat ». Kanagaretnam et al. (2005) testent la pertinence des composantes du CI sur un échantillon d'entreprises canadiennes entre 1998 et 2002. Ils concluent positivement, mais relèvent que le résultat net a une vertu prédictive supérieure à toutes les autres composantes du CI. Chambers et al. (2006) montrent que l'adoption de FAS 
130 en 1997 a renforcé de façon significative, au regard de la période antérieure, la valuerelevance de certains OCI (notamment ceux qui concernent les portefeuilles de placement comptabilisés en juste valeur). Cependant, ces travaux ne mettent en évidence aucun effet significatif de la divulgation du comprehensive income sous la forme d'un compte de résultat agrégeant net income et OCI.

\subsection{Quel contenu informatif pour le comprehensive income en Europe?}

Les travaux empiriques antérieurs n’ont pas permis de démontrer, dans un contexte se référant essentiellement au cadre normatif nord-américain, la suprématie du comprehensive income sur le résultat net en termes de degré de pertinence et d'utilité informationnelle. La présente analyse se propose de comparer, dans un contexte européen — plus particulièrement français et britannique —, la value-relevance et l'utilité des mesures du résultat qui sont au cœur du débat relatif au projet Performance Reporting de l’IASB. Cette étude empirique, réalisée dans un cadre réglementaire et sur des marchés européens, devrait permettre de répondre aux questions de recherche suivante :

Q1 : Le comprehensive income, agrégeant net income et other comprehensive income, est-il plus informatif en termes de degré de pertinence que les mesures traditionnelles du résultat (i.e. résultat net et résultat opérationnel) ?

Q2 : Les other comprehensive income ajoutent-ils de l'information à celle déjà véhiculée par le résultat net ou le résultat opérationnel ? 


\subsection{Méthodologie de recherche}

Nous présenterons successivement la méthode de construction des variables proxies, puis le modèle testable qui sera décliné pour chacune des métriques de résultat (Ramond, 2006).

\subsubsection{Construction des variables et mesure du comprehensive income}

En Europe, les cadres réglementaires nationaux et les pratiques de divulgation étant très contrastés, l'utilisation de dirty surplus varie significativement d'un pays à l'autre (nondivulgation du comprehensive income en France vs. enracinement du concept au RoyaumeUni). En vue de faciliter la comparaison des deux pays échantillonnés, nous avons construit un proxy du dirty surplus flow agrégé inspiré de la relation de clean surplus proposée par Ohlson (1991). L'articulation entre les montants du bilan, les flux comptables, les dividendes et les variations de capital pour chaque période financière, $t$, est définie comme suit ${ }^{6}$ :

$$
B V_{t} \equiv B V_{t-1}+N_{t}-d_{t}+C I_{t}
$$

Ou encore :

$$
C I_{t} \equiv \Delta B V_{t}-N_{t}+d_{t}
$$

en notant $\Delta$, les variations entre deux périodes financières $t-1$ à $t ; B V$, les capitaux propres (book value) ; $N$, le montant total des titres émis ; $d$, le montant des dividendes annuels et $C I$, le comprehensive income de l'entreprise.

Le CI étant défini comme un résultat global (all-inclusive income), il est alors possible d'écrire :

$$
C I_{t} \equiv N I_{t}+D S_{t}
$$


En notant $N I$, le résultat net (net income ou bottom-line income) de l'entreprise et $D S$, le montant du dirty surplus earnings (ou other comprehensive income).

\subsubsection{Proposition d'un modèle de value-relevance du résultat}

L’idée selon laquelle la valeur comptable des capitaux propres et la valeur de marché sont intimement liées, comme deux composantes — nominale et vénale — de la valeur d'une action, a été largement relayée dans la littérature financière au cours de ces deux dernières décennies (e.g. Harris et Ohlson (1987) ; Lev (1989) ; Easton et Harris (1991)). Cependant, les liens qui ont été observés entre le prix de l’action, les bénéfices, le flux de dirty surplus et les autres mouvements de capitaux propres n’ont été que rarement démontrés de manière formelle. Dans un cadre formel d'analyse, Ohlson (1995) démontre la relation suivante :

$$
P_{t}=k\left(\varphi C I_{t}-d_{t}\right)+(1-k) B V_{t}+\alpha V_{t}
$$

En notant $P$, le prix d'une action de l'entreprise à une date t ; CI, le comprehensive income par action ; $d$, les dividendes annuels par action ; $V$, l’information additionnelle sur les résultats futurs qui n’est reflétée ni dans les résultats présents, ni dans la book value de la période actuelle $t$; $k$, un facteur de pondération de la contribution des variations de la book value, i.e. $\phi C I-d$, (par rapport à la book value elle-même) dans l'explication de la valeur des actions ; et $\phi$ et $\alpha$, d'autres paramètres estimés.

Ce modèle d'évaluation établit que la valeur d'une entreprise est une simple pondération entre un modèle patrimonial (book value model), i.e. $B V_{t}$, et un modèle de rente (earnings model), i.e. $\varphi C I_{t}-d_{t}$. La littérature sur les relations rendement-résultat (e.g. Easton et Harris (1991) ; O’Hanlon et Pope (1999)) a souvent étudié une forme économétrique simplifiée du modèle précédent :

$$
P_{t}=\alpha_{0}+\alpha_{1} B V_{t}+\alpha_{2} C I_{t}+\varepsilon_{t}
$$


En notant $P$, le prix de l'action ajusté pour les dividendes ; $B V$, la book value par action de l'entreprise; CI, le comprehensive income (ou bénéfices) par titre et l'indice $t$ la période comptable d'analyse.

Afin d'éviter un effet d'échelle sur les résultats statistiques inhérent à toute régression prixrésultat, les auteurs (e.g. Cheng et al. (1993) ; Easton (1999)) proposent classiquement de tester un autre modèle qui transforme les variables dépendantes - le prix des titres $P$ — en une variable de rendement des titres. Le modèle (3) est reformulé comme suit :

$$
R E T_{t}=\beta_{0}+\beta_{1} \frac{C I_{t}}{P_{t-1}}+\beta_{2} \frac{\Delta C I_{t}}{P_{t-1}}+u_{t}
$$

avec $R E T$, le rendement cumulé moyen des titres d'une entreprise ${ }^{7} ; P$, le prix de l'action ajusté pour les dividendes; $C I$, le comprehensive income par titre ; $\Delta C I$, la variation du comprehensive income par titre et $t$, la période comptable d'analyse.

En se basant sur (1c), nous pouvons reformuler (4) ainsi :

$$
R E T_{t}=\gamma_{0}+\gamma_{1} \frac{N I_{t}}{P_{t-1}}+\gamma_{2} \frac{\Delta N I_{t}}{P_{t-1}}+\gamma_{3} \frac{D S_{t}}{P_{t-1}}+\gamma_{4} \frac{\Delta D S_{t}}{P_{t-1}}+\omega_{t}
$$

\subsubsection{Déclinaison du modèle pour chaque métrique du résultat}

L’étude empirique repose sur la déclinaison du modèle de base ci-dessus en fonction de chaque métrique de résultat. Selon la méthodologie utilisée par Cheng et al. (1993) et Lin (2006), le contenu informatif des différentes mesures de résultat est empiriquement étudiée, selon la structure classique d'un compte de résultat, à partir du modèle (5). Cette approche conduit à tester empiriquement, pour chacun des deux pays échantillonnés, les quatre sousmodèles suivants :

Modèle du résultat opérationnel :

$$
R E T_{t}=\gamma_{0}^{\prime}+\gamma_{1}^{\prime} \frac{O P I N_{t}}{P_{t-1}}+\gamma_{2}^{\prime} \frac{\Delta O P I N_{t}}{P_{t-1}}+\omega_{t}^{\prime}
$$


Modèle du résultat net :

$$
R E T_{t}=\gamma_{0}^{\prime \prime}+\gamma_{1}^{\prime \prime} \frac{N I_{t}}{P_{t-1}}+\gamma_{2}^{\prime \prime} \frac{\Delta N I_{t}}{P_{t-1}}+\omega_{t}^{\prime \prime}
$$

Modèle du comprehensive income :

$$
R E T_{t}=\gamma_{0}^{(3)}+\gamma_{1}^{(3)} \frac{C I_{t}}{P_{t-1}}+\gamma_{2}^{(3)} \frac{\Delta C I_{t}}{P_{t-1}}+\omega_{t}^{(3)}
$$

Modèle des other comprehensive income :

$$
R E T_{t}=\gamma_{0}^{(4)}+\gamma_{1}^{(4)} \frac{N I_{t}}{P_{t-1}}+\gamma_{2}^{(4)} \frac{\Delta N I_{t}}{P_{t-1}}+\gamma_{3}^{(4)} \frac{D S_{t}}{P_{t-1}}+\gamma_{3}^{(4)} \frac{\Delta D S_{t}}{P_{t-1}}+\omega_{t}^{(4)}
$$

\section{Echantillon et collecte des données}

Les données ont été collectées à partir des bases DATASTREAM et WORLDSCOPE, sur la période 1992-2004, pour les entreprises cotées au Royaume-Uni et en France ${ }^{8}$. Les normes IAS / IFRS étant entrées en vigueur au $1^{\mathrm{er}}$ janvier 2005, nous n’avons pas inclus cette année de transition dans l'échantillon, les comptes ainsi publiés pouvant biaiser les résultats statistiques ${ }^{9}$.

Lors de l'utilisation de la classification industrielle de DATASTREAM au niveau 6, code INDC $6^{10}$, nous avons éliminé les observations portant sur les codes 8000 à 8999, relatives aux sociétés financières dont les pratiques de divulgation sont différentes. Nous avons focalisé l'échantillon sur les entreprises industrielles afin d'obtenir des résultats plus robustes et dans un souci de cohérence avec le projet de l'IASB ${ }^{11}$. 
Durant la période d’observation, le comprehensive income n’étant pas divulgué dans les états financiers en France, les bases DATASTREAM et WORLDSCOPE ne proposent aucune donnée directement utilisable. Cependant, tous les éléments nécessaires au calcul étant disponibles dans le tableau de variation des capitaux propres des entreprises analysées, nous avons procédé à la détermination du comprehensive income en utilisant la formule du cleansurplus :

$$
C I_{t} \equiv \Delta B V_{t}+d_{t}-N_{t}
$$

en notant $B V$, la valeur comptable des capitaux propres ; $N$, les nouveaux titres émis et $d$, les dividendes versés pendant la période financière d'observation. Les entreprises dont les données comptables (valeur comptable des capitaux propres) n’étaient pas disponibles ont été éliminées de l'échantillon. Conformément aux pratiques retenues par des études antérieures (e.g. O’Hanlon et Pope, 1999 ; Lin, 2006), les observations manquantes pour les variables « Nouveaux titres émis» et «Dividendes » ont été remplacées par une valeur nulle ${ }^{12}$. Par ailleurs, toute firme dont les données financières n’étaient pas disponibles sur DATASTREAM a été exclue de l'échantillon. Enfin, afin d'éviter les problèmes potentiels causés par les observations extrêmes (outliers), les observations portant sur les centiles extrêmes (i.e. 1\% et 99\%) de la distribution de chaque variable ont été éliminées de l'analyse. Le tableau 2 présente la composition de l'échantillon par pays, avant et après suppression des outliers, pour chacun des modèles testés.

INSERER LE TABLEAU 2

\section{Value-relevance et utilité des différentes mesures du résultat}

\subsection{Statistiques univariées et bivariées}


Les statistiques relatives aux variables d'analyse sont présentées dans le tableau 3. Les variables comptables sont toutes exprimées sur une base unitaire (i.e. par action) et sont divisées par le cours de l'action de début de période. Le Panel A montre que la moyenne (médiane) du CI est respectivement de -0.010 (0.041) au Royaume-Uni, et de 0.032 (0.049) en France. La valeur moyenne (médiane) du résultat net est de -0.021 (0.049) au Royaume-Uni et de 0.033 (0.052) en France. Le résultat net est donc inférieur, en moyenne, au CI au Royaume-Uni, mais pas en France ${ }^{13}$. Le test bilatéral de Mann-Whitney-Wilcoxon montre que le CI et le résultat net sont significativement différents dans tous les échantillons. A la suite de ce constat, nous choisissons d'examiner la distribution de la variable du dirty surplus flow (DSF) dans ces deux échantillons. Le test de Student (non communiqué) indique que les variables DSF au Royaume-Uni et en France sont différentes de zéro à un niveau de confiance de 99\%. Par conséquent, nous nous attendons à ce que les variables $N I$ et $C I$ fournissent des degrés de pertinence distincts dans les deux pays échantillonnés.

Le panel B présente les coefficients de corrélation de Pearson et Spearman (méthode des rangs) pour toutes les variables indépendantes du modèle. Comme pour le panel $\mathrm{A}$, les statistiques sont présentées par pays. Les résultats indiquent que le flux de dirty surplus (DSF) présente (1) une corrélation significative et négative avec le résultat net (NI) et le résultat d'exploitation $(O P I)$ dans tous les échantillons ${ }^{14}$ et (2) une corrélation positive et significative avec le comprehensive income $(C I)$ et la variation du comprehensive income $(\Delta C I)$.

\section{[INSERER LE TABLEAU 3]}

\subsection{Degré de pertinence des composants de la performance en France et au Royaume-Uni}

Les statistiques de Shapiro-Wilks (non communiquées) indiquent que les composants de la performance ne sont pas distribués normalement dans nos échantillons. Les observations extrêmes pourraient influencer les statistiques de la méthode conventionnelle des moindres- 
carrés (MCO). Pour pallier ce problème, deux techniques de régression ont été utilisées, permettant d'effectuer une comparaison et un test de robustesse des résultats statistiques entre eux : (1) utilisation des éléments de performance comptable comme variable indépendante dans l'approche MCO conventionnelle ; (2) régression MCO fondée sur la méthode des rangs des mesures du résultat comme variable indépendante (notée ci-après la méthode des rangs). Elle a été utilisée dans de nombreuses études empiriques (cf. Abarbanell \& Bushee, 1988 ; Raedy, 2000 ; Lin, 2006) pour standardiser toutes les variables explicatives afin : (1) de réduire l'impact d'observations extrêmes potentielles et (2) de prendre en compte les relations non linéaires potentielles existantes entre les rendements des titres et les données comptables. Les résultats relatifs à ces deux approches sont présentés dans le tableau 4.

\section{[INSERER LE TABLEAU 4]}

\subsubsection{Degré de pertinence du résultat opérationnel}

Le panel A du tableau 4 présente les résultats de la régression de la value-relevance du résultat opérationnel dans les pays européens. Les résultats indiquent que le coefficient de pente du niveau et de la variation du résultat opérationnel (respectivement $O P I$ et $\triangle O P I$ ) sont pertinents quelque soit l'échantillon considéré. Cependant, l'utilité de cet élément pour les investisseurs (tel qu’approximé par la statistique $R^{2}$-ajusté de la régression) varie considérablement d'un échantillon à l'autre. Ainsi, le résultat d'exploitation décrirait mieux le rendement des titres dans un pays latin comme la France. Ces résultats sont confirmés par la méthode des rangs. En effet, (1) les statistiques $R^{2}$ selon cette approche sont bien plus élevées que celles obtenues en utilisant la méthode conventionnelle des MCO, ce qui indiquerait que la méthode des rangs convient mieux à la relation entre le rendement des titres et les composants du résultat; (2) $O P I$ et $\triangle O P I$ sont tous deux statistiquement significatifs au 
niveau de signification de $1 \%$ ce qui suggère que le résultat opérationnel est pertinent dans tous les pays échantillonnés ; (3) les coefficients de pente sont sensiblement plus faibles que ceux obtenus en utilisant la méthode conventionnelle des MCO, il semblerait donc que la méthode des rangs diminue l'influence des observations extrêmes (outliers) ; (4) la statistique $R^{2}$ est plus élevée dans l'échantillon français (16.59\%), ce qui prouve, suivant le cadre d’analyse de Lev (1989), que le résultat opérationnel est plus « utile » dans ce pays qu’au Royaume-Uni (11.55\%).

\subsubsection{Degré de pertinence du résultat net}

Le panel B du tableau 4 présente les résultats de la value-relevance du résultat net selon la méthode conventionnelle des MCO (Panel A) et selon la méthode des rangs (Panel B). Le résultat net est associé, de manière significative, au rendement des titres dans tous les échantillons. D’après la méthode conventionnelle des MCO, le niveau et les variations du résultat net sont statistiquement significatifs à un niveau de confiance de 99\% (95\%) en France (au Royaume-Uni). Par ailleurs, les statistiques $R^{2}$ de MCO sont sensiblement plus élevées dans l'échantillon français, ce qui suggère que le résultat net est plus « utile » dans cet environnement financier qu'au Royaume-Uni.

Les valeurs $R^{2}$ du modèle (6b) obtenues à partir de la méthode des rangs (10.42\% pour le Royaume-Uni et $23.68 \%$ pour la France) sont bien plus élevées que celles trouvées avec la méthode conventionnelle des MCO (4.88\% pour le Royaume-Uni et 13.60\% pour la France) ; (2) les coefficients de pente des variations et du niveau du résultat (respectivement, $N I$ et $\Delta N I$ ) sont bien moins élevés que ceux estimés par la méthode conventionnelle des MCO, ce qui semble indiquer la présentation d'outliers ; (3) NI et $\Delta N I$ semblent être associés, de manière positive, avec le rendement des titres à un niveau de signification de $1 \%$ dans les deux pays. La méthode des rangs semble donc mieux convenir à l'étude de l'association des rendements 
des titres et du résultat net, notamment en réduisant l'impact des observations extrêmes sur les estimateurs de la régression des MCOs.

Par ailleurs, quelque soit la méthode de régression utilisée, le Royaume-Uni présente une statistique $R^{2}$ bien plus faible que la France, ce qui laisserait penser que le résultat net fournit moins d’informations aux investisseurs dans cet environnement financier.

\subsubsection{Degré de pertinence du comprehensive income}

Le panel C présente les résultats du degré de pertinence du comprehensive income par pays. Le niveau et les variations du comprehensive income (respectivement $C I$ et $\Delta C I$ ) sont tous deux associés positivement et statistiquement, au moins à un niveau de confiance de 99\%, au rendement des titres (à l'exception de $\Delta C I$ au Royaume-Uni, où cette variable n'est pas significative selon la méthode des rangs). Nous trouvons encore que les valeurs de la statistique $R^{2}$ obtenues avec la méthode des rangs (6.38\% pour le Royaume-Uni et $14.92 \%$ pour la France) sont bien plus élevées que celles de la méthode conventionnelle (respectivement de $3.57 \%$ et $7.64 \%$ ). Par ailleurs, la statistique $R^{2}$ est plus élevée en en France qu’au Royaume-Uni, ce qui suggère une plus grande «utilité » du comprehensive income dans l'environnement français.

En résumé, le tableau 4 met empiriquement en évidence que le résultat opérationnel, le résultat net et le comprehensive income sont tous statistiquement associés au rendement du titre, tant en France qu’au Royaume-Uni. Néanmoins, il apparaît que le résultat net et le comprehensive income sont plus utiles - tels que mesuré par la statistique $R^{2}$ — pour les investisseurs intervenant sur la place financière française. Par ailleurs, il est intéressant de noter que les investisseurs britanniques semblent ne pas valoriser autant que leurs homologues français les deux mesures de résultat : le résultat net et le comprehensive income. Ils semblent se concentrer plutôt sur la mesure du résultat opérationnel. Ces résultats suggèrent que le 
résultat net et le comprehensive income sont plus utiles aux investisseurs français qu'aux investisseurs britanniques. Le résultat opérationnel semblerait être la mesure la plus utilisée dans le cas du Royaume-Uni. Ce résultat est cohérent avec celui discuté dans l'étude de Lin (2006). En effet, utilisant un échantillon d'entreprises britanniques sur la période post-FRS 3 (1993-1998), Lin (2006) trouve que le résultat opérationnel fournit une information additionnelle par rapport au résultat avant impôt. Ce résultat est également cohérent avec ceux exposés dans l'étude américaine de Cheng et al. (1993) qui trouvent que le résultat opérationnel est associé plus fortement avec le rendement des titres que le résultat net et le comprehensive income.

\subsubsection{Information additionnelle délivrée par les OCI}

Le panel $\mathrm{D}$ du tableau 4 présente les résultats relatifs au degré de pertinence additionnel du dirty surplus flow par rapport à la mesure du résultat net. Le panel A montre que le niveau de dirty surplus est négativement (mais pas significativement) associé au rendement des titres en France (au Royaume-Uni), mais que la variation du dirty surplus flow ( $\triangle D S F$ ) est positivement associée au rendement des titres dans les deux pays. De nouveau, nous trouvons que le $R^{2}$ est plus élevé en France. Selon la méthode des rangs, les coefficients de pente du niveau et de la variation du dirty surplus flow sont significatifs, aux niveaux conventionnels, après avoir contrôlé pour l'effet dû au résultat net. La France présente un $R^{2}$ beaucoup plus élevé que le Royaume-Uni.

En résumé, nous observons empiriquement que le dirty surplus flow est pertinent, dans les deux pays échantillonnés, et qu’il véhicule une information additionnelle par rapport au résultat net. Il en est de même pour la variation de dirty surplus flow. Nos résultats montrent 
également que les éléments composant le dirty surplus sont généralement plus utiles pour les investisseurs en France qu'au Royaume-Uni.

Afin de déterminer si le comprehensive income est plus pertinent que le résultat d'exploitation et le résultat net, nous avons procédé à un test non-imbriqué de Vuong (1989) pour comparer l'utilité du modèle (6c) avec celles des modèles (6a) et (6b). Le panel B (tableau 5) présente les résultats du test: la statistique-z de Vuong montre que le comprehensive income fait preuve de moins de pertinence que le résultat net à un niveau de signification de $1 \%$ dans les deux pays. Par ailleurs, les statistiques de Vuong indiquent également une pertinence moindre du comprehensive income par rapport au résultat d'exploitation, au seuil de signification conventionnel. Ce dernier résultat, combiné avec les précédents, nous permet de conclure que le résultat net est le plus utile des agrégats de performance comptable en France. Les résultats empiriques indiquent également que le résultat opérationnel est plus pertinent au RoyaumeUni que le résultat net et le comprehensive income. Ce résultat intéressant pourrait être expliqué par le fait que le résultat opérationnel doit être obligatoirement divulgué par les sociétés cotées depuis l’entrée en vigueur de la norme FRS 3 (qui a standardisé la divulgation de cette mesure). Au contraire, les investisseurs français ne sont pas forcément familiers avec cette mesure qui n’est définie clairement dans aucune norme locale.

\section{[INSERER LE TABLEAU 5]}

\subsubsection{Comparaison avec les études antérieures}

Les résultats précédemment discutés sont contraires à ceux obtenus par Cheng et al. (1993), Dhaliwal et al. (1999), O’Hanlon et Pope (1999) et Chambers et al. (2006) à partir de données nord-américaines et britanniques. Ces travaux observent que la variable DSF n'est pas valorisée (au sens de pricing) durant la période pré-SFAS 130, i.e. lorsqu'elle n’est pas 
clairement divulguée dans les états financiers. En revanche, nos résultats, relatifs au contexte européen, sont tout à fait cohérents avec ceux mis en évidence par Kanagaretnam et al. (2005), Biddle et Choi (2006) et par Lin (2006).

\section{Conclusion}

Cette étude examine les caractéristiques informationnelles, en termes de value-relevance et d’utilité, dont font preuve, sur les marchés financiers britannique et français, les trois mesures du résultat comptable — résultat net, opérationnel et comprehensive income — objet du projet Performance Reporting commun à l'IASB et au FASB. Nos résultats montrent que ces trois mesures du résultat comptable sont associées de manière significative au rendement boursier d’une entreprise sur chacun des marchés, tout en faisant preuve de degrés de value-relevance et d'utilité variés. De manière plus précise, nous trouvons que le comprehensive income, en variable agrégée, fournit moins d’information que le résultat net, tant en France et qu’au Royaume-Uni. Néanmoins, dans nos échantillons, les other comprehensive income (OCI) véhiculent, dans les deux environnements financiers, une information additionnelle par rapport à celle qui est apportée par le résultats net. Bien que ce contenu informatif des OCI milite en faveur de leur divulgation dans les états financiers, ces résultats vont tout de même à l'encontre de l’idéologie sous-jacente au projet de l'IASB qui vise à remplacer le résultat net par le comprehensive income. Les conclusions de la présene étude sont cependant à replacer dans le contexte de la révision en cours du cadre conceptuel de l’IASB — projet qui doit redéfinir les caractéristiques qualitatives de l'information comptable, les catégories d'utilisateurs ainsi que leurs besoins. Ce futur cadre conceptuel apportera, sans nul doute, de nouveaux arguments pour alimenter le débat sur l’avenir du « résultat net ». 


\section{Bibliographie}

Abarbanell, J.S. et B.J. BusheE (1988), «Abnormal Returns to a Fundamental Analysis Strategy », The Accounting Review, Janvier, 73(1), pp.19-45.

AcCounting StANDARDS BoARD (1992), «Financial Reporting Standard N³: Reporting Financial Performance », IAS Publications, Octobre, London, UK.

AMIR, E., T.S. HARRIS et E.K. VeUTI (1993), « A Comparison of the Value-Relevance of US vs. Non-US GAAP Accounting Measures Using Form 20-F Reconciliations », Journal of Accounting Research, 31, pp.230-64.

Association FOR InVESTMENT MANAGEMENT AND RESEARCH (AIMR) (1993), " Financial Reporting in the 1990's and Beyond », Charlottesville, Virginia: AIMR.

BALL, R. (1995), « Making Accounting More International: Why, How, and How Far Will It Go? », Journal of Applied Corporate Finance, Automne, 8(3), pp.19-29.

BARKer, R. (2003), « Global Accounting Is Coming », Harvard Business Review, Avril, pp.24-5.

BArker, R. (2004), « Reporting Financial Performance », Accounting Horizons, 18(2), pp.157-72.

BEAVER, W. (1981), « Financial Reporting: an Accounting Revolution », Prentice-Hall Contemporary, Englewood Cliffs, N.J.: Prentice-Hall.

BIDDle, G. et J.H. CHOI (2006), « Is Comprehensive income Useful? », Journal of Contemporary Accounting and Economics, 2(1), Juin, à paraître.

BlacK, F. (1980), « The Magic in Earnings: Economic Earnings vs. Accounting Earnings », Financial Analysts Journal, Novembre-Décembre, pp.19-24.

BlacK, F. (1993), « Choosing Accounting Rules », Accounting Horizons, 7, pp.1-17.

Brief, R. P. et K.V. PeAsnell (eds.) (1996), « Clean Surplus: a Link between Accounting and Finance », New York: Garland Publishing.

Bushman, R.M. et A.J. Smith (2001), « Financial Accounting Information and Corporate Governance », Journal of Accounting and Economics, 32, pp.237-333.

CASTA, J-F. et B. COLASSE (eds.) (2001), « Juste valeur : enjeux techniques et politiques », Ed. Economica.

Chambers, D., T.J. LinsmeiER, C. ShaKespeAre et T. Sougiannis (2006), « An Evaluation of SFAS n¹30 Comprehensive income Disclosures », Review of Accounting Research, à paraître.

Chartered Financial Analysts (CFA) Institute (2005), « A Comprehensive Business Reporting Model: Financial Reporting for Investors », Octobre, Charlottesville, Virginia, 66p.

Cheng, A., J. Cheung et V. Gopalakrishnan (1993), « On the Usefulness of Operating Income, Net Income and Comprehensive income in Explaining Security Returns », Accounting and Business Research, 23, n91, pp.195-203.

Collins, D.W., MAYdew, E.L. et Weiss, I.S. (1997), « Changes in the value-relevance of earniongs and book values over the past forty years », Journal of Accounting and Economics, Vol.24, Issue 1, 39-67.

Cope, A.T., L.T. Johnson et C.L. Reither (1996), « The Call for Reporting Comprehensive income », Financial Analysts Journal, Mars/Avril, 52(2), pp.7-12.

Dhaliwal, D., K. Subramnayam et R. Trezevant (1999), « Is Comprehensive income Superior to Net Income as a Measure of Firm Performance? », Journal of Accounting and Economics, 26, pp.43-67. 
EAston, P. (1999), "Security Returns and Value Relevance of Accounting Data », Accounting Horizons, Décembre, 3, pp.399-412.

EASTON, P. et T. HARRIS (1991), « Earnings as an Explanatory Variable for Returns », Journal of Accounting Research, Printemps, pp.19-36.

ELy, K. et WAyMiRE, G. (1996), « Accounting Standard-Setting Organizations and Earnings Relevance : Longitudinal Evidence from NYSE Common Stocks, 1927-93 », Journal of accounting Research, Vol.37, $\mathrm{n}^{\circ} 2: 293-317$.

FinANCiAl AcCounting StAndARDS BoARD (1997), « Statement of Financial Accounting Standard N¹30: Reporting Comprehensive income », FASB, Juin 1997.

FrANCIS, J. et SCHIPPER, K. (1999), « Have Financial Statements Lost Their Relevance ? », Journal of accounting Research, Vol.37, n² :319-52.

HARRIS, T. et J. OHLsOn (1987), « Accounting Disclosures and the Market Valuation of Oil and Gas Properties », The Accounting Review, Octobre, pp.651-70.

Hirst, E., et Hopkins, P. (1998), "Comprehensive income reporting and analysts’ valuation judgments », Journal of Accounting Research, 36 (Supplément), pp.47-75.

InTERnAtional AcCounting StAndARDs BoARd (IASB) (2006), « Exposure Draft of Proposed Amendments to IAS 1 Presentation of Financial Statements », IASCF, Mars, 103p.

Kanagaretnam, K., R. MAthiEU et M. ShehatA (2005), « Usefulness of Comprehensive income Reporting in Canada », Working Paper, Février, McMaster University, Ontario, Canada.

Kiger, J. E. et J.R. WiLliams (1977), « An Emerging Concept of Income Presentation », Accounting Historians Journal, 4 (2), pp.63-77.

Leuz, C. et R.E. Verrecchia (2000), « The Economic Consequences of Increased Disclosure’, Journal of Accounting Research, 38 (Supplément), pp.91-124.

LEV, B. (1989), « On the Usefulness of Earnings and Earnings Research: Lessons and Directions from Two Decades of Empirical Research », Journal of Accounting Research, Supplément, pp.153-92.

LIN, S. (2006), « Testing the Information Set Perspective of UK Financial Reporting Standard N³: Reporting Financial Performance », Journal of Business, Finance and Auditing, à paraître.

Linsmeier, T.J., Gribble, R., Jennings R.G., Lang, M.H., Penman, S.H., Petroni, K.R., Shores, D., Smith, J.H. et WARFIELD, T.D. (1997), « An Issues Paper on Comprehensive income », Accounting Horizons, Juin, 11(2), pp.120-6.

O’Hanlon, J. et P. Pope (1999), « The Value Relevance of U.K. Dirty surplus Accounting Flows’ », British Accounting Review, 31, 459-482.

OHLson, J.A. (1989), « Accounting Earnings, Book Value, and Dividends: The Theory of the Clean Surplus Equation », Unpublished Paper, Columbia University, USA.

OhLson, J.A. (1991), « The Theory of Value and Earnings, and an Introduction to the Ball-Brown Analysis », Contemporary Accounting Research, 7, pp.1-19.

Ohlson, J.A. (1995), « Earnings, Book Values, and Dividends in Equity Valuation », Contemporary Accounting Research, 11(2), pp.661-78.

OHLson, J.A. (1999), « Earnings, Book Values, and Dividends in a Stewardship Setting with Moral Hazard », Contemporary Accounting Research, 16 (3), pp.525-40. 
Pope P.F. et P. WANG (2005), « Earnings Components, Accounting Bias and Equity Valuation », Review of Accounting Studies, 10, pp.387-407.

RAEDY, J. (2000), « A Reconciliation of Stock Market Anomalies », Working paper, University of North Carolina, Chapel Hill.

RAMOND, O. (2006), « Contribution à l'étude du degré de pertinence de différentes mesures du résultat : résultat net, opérationnel et comprehensive income », Thèse de doctorat en sciences de gestion, Université Paris Dauphine.

Robinson, L.E. (1991), « The Time Has Come to Report Comprehensive income », Accounting Horizons, 5(2), pp.107-12.

VuONG, Q.H. (1989), « Likelihood Ratio Tests for Model Selection and Non-nested Hypotheses », Econometrica, Mars, 57(2), pp.307-33.

ZEFF, S.A. (1978), « The Rise of Economic Consequences », The Journal of Accountancy, Décembre, pp.56-63. 
Tableau 1. Principaux postes comptables inscrits au bilan et contournant le compte de résultat (dirty surplus items) en normes françaises, US, UK et IAS / IFRS

\begin{tabular}{|c|c|c|c|c|}
\hline Postes de « dirty surplus » & French GAAPs & US GAAPs & UK GAAPs & IAS / IFRS \\
\hline Réévaluation des actifs & CRC 99-02 (*) & N/A & FRS $15(*)$ & IAS $16(*)$ \\
\hline $\begin{array}{l}\text { Ecarts de conversion des } \\
\text { investissements nets }\end{array}$ & CRC 99-02 (*) & FAS $52(*)$ & SSAP $20(*)$ & IAS $21(*)$ \\
\hline $\begin{array}{l}\text { Ajustement des provisions sociales } \\
\text { / départ à la retraite }\end{array}$ & N/A & FAS $87(*)$ & N/A & IAS $19(*)$ \\
\hline $\begin{array}{l}\text { Pertes et gains latents sur des } \\
\text { instruments financiers de trésorerie } \\
\text { (ou liquides) }\end{array}$ & N/A & FAS $115(*)$ & FRS $13(*)$ & IAS $39(*)$ \\
\hline $\begin{array}{l}\text { Pertes et gains latents sur } \\
\text { instruments dérivés }\end{array}$ & N/A & FAS $133(*)$ & $\begin{array}{l}\text { FRS } 25 \text { et } \\
\text { FRS } 26(*)\end{array}$ & IAS $39(*)$ \\
\hline $\begin{array}{l}\text { Traitement des écarts d'acquisition } \\
\text { (ou goodwill acquis) }\end{array}$ & $\begin{array}{l}\text { CRC 99-02 } \\
\text { (art. } 212 \text { et 215) }\end{array}$ & N/A & $\begin{array}{l}\text { ex-SSAP } 22 \\
\text { et FRS } 10(*)\end{array}$ & N/A \\
\hline $\begin{array}{l}\text { Postes concernant les changements } \\
\text { de méthodes comptables }\end{array}$ & $\begin{array}{l}\text { 314-1 et } 314-2 \\
\text { du PCG }(*)\end{array}$ & $\begin{array}{c}\text { APB } 20 \\
\text { et FAS } 11(*)\end{array}$ & N/A & N/A \\
\hline Provisions réglementées & CRC 99-02 (*) & N/A & N/A & N/A \\
\hline Divers postes d'impôts et taxes & N/A & FAS $109(*)$ & N/A & N/A \\
\hline $\begin{array}{l}\text { Dividendes prioritaires et pertes } \\
\text { sur rachat des actions à dividendes } \\
\text { prioritaires }\end{array}$ & N/A & $(*)$ & N/A & N/A \\
\hline
\end{tabular}

Le symbole (*) indique que le poste comptable concerné contourne le compte de résultat. Sources : Barker (2004) et Ramond (2006). 
Tableau 2. Modèles des composants du résultat (earnings components) et sélection des échantillons d'analyse

\begin{tabular}{|l|c|c|}
\cline { 2 - 3 } \multicolumn{1}{c|}{} & \multicolumn{2}{c|}{ Nombre d'observations } \\
\cline { 2 - 3 } \multicolumn{1}{c|}{} & $\begin{array}{c}\text { Avant la } \\
\text { suppression } \\
\text { des outliers }\end{array}$ & $\begin{array}{c}\text { Après la } \\
\text { suppression } \\
\text { des outliers }\end{array}$ \\
\hline Model (6a) : Résultat opérationne & & \\
- Royaume-Uni & 8,183 & 7,915 \\
- France & 4,249 & 4,123 \\
Model (6b) : Résultat net & & \\
- Royaume-Uni & 8,165 & 6,983 \\
- France & 4,017 & 3,564 \\
Model (6c) : Comprehensive Income & & \\
- Royaume-Uni & 7,403 & 7,186 \\
- France & 3,987 & 3,865 \\
Model (6d) : Résultat net et Dirty Surplus Flow & & \\
- Royaume-Uni & 7,340 & 6,983 \\
- France & 3,750 & 3,564 \\
\hline
\end{tabular}


Tableau 3. Statistiques univariées pour les variables utilisées dans l'étude

Panel A. Statistiques univariées pour les variables dépendantes et indépendantes

\begin{tabular}{|c|c|c|c|c|c|c|}
\hline Variable & $\mathrm{N}$ & Moyenne & Médiane & Ecart-type & Asymétrie & Kurtosis \\
\hline \multicolumn{7}{|l|}{ Royaume-Uni } \\
\hline Rendement du titre (\%) & 7,895 & 0.034 & 0.051 & 0.289 & -0.480 & 1.296 \\
\hline Comprehensive Income & 8,031 & $-0.010^{* * *}$ & $0.041^{* * *}$ & 0.247 & -2.407 & 11.256 \\
\hline Variation du Comprehensive Income & 7,151 & 0.028 & 0.008 & 0.338 & 2.461 & 18.312 \\
\hline Dirty Surplus Flow & 8,031 & 0.012 & -0.001 & 0.134 & 2.711 & 18.235 \\
\hline Variation du Dirty Surplus Flow & 7,136 & 0.002 & 0.000 & 0.193 & 0.928 & 14.577 \\
\hline Résultat net & 8,031 & -0.021 & 0.049 & 0.245 & -3.352 & 14.443 \\
\hline Variation du Résultat net & 7,992 & 0.021 & 0.008 & 0.278 & 2.644 & 21.749 \\
\hline Résultat opérationnel & 7,934 & $0.053^{* * *}$ & $0.085^{* * *}$ & 0.171 & -1.978 & 7.367 \\
\hline Variation du Résultat opérationnel & 7,896 & 0.015 & 0.010 & 0.124 & 1.571 & 11.840 \\
\hline \multicolumn{7}{|l|}{ France } \\
\hline Rendement du titre (\%) & 3,888 & 0.106 & 0.116 & 0.462 & -0.273 & 0.539 \\
\hline Comprehensive Income & 3,963 & $0.032 * * *$ & $0.049 * * *$ & 0.152 & -1.617 & 7.954 \\
\hline Variation du Comprehensive Income & 3,673 & 0.007 & 0.004 & 0.192 & 1.336 & 13.251 \\
\hline Dirty Surplus Flow & 3,963 & -0.001 & 0.000 & 0.069 & 1.338 & 17.761 \\
\hline Variation du Dirty Surplus Flow & 3,663 & -0.007 & 0.000 & 0.103 & -0.701 & 13.419 \\
\hline Résultat net & 3,963 & 0.033 & 0.052 & 0.142 & -2.137 & 9.785 \\
\hline Variation du Résultat net & 3,910 & 0.015 & 0.006 & 0.146 & 2.325 & 17.225 \\
\hline Résultat opérationnel & 3,940 & $0.079 * * *$ & $0.082^{* * *}$ & 0.162 & -0.544 & 4.589 \\
\hline Variation du Résultat opérationnel & 3,913 & 0.014 & 0.008 & 0.124 & 1.171 & 8.273 \\
\hline
\end{tabular}

Panel B. Coefficients de corrélation de Pearson/Spearman

Echantillon britannique $(N=6,946)$

\begin{tabular}{|c|c|c|c|c|c|c|c|c|}
\hline & $\mathrm{CI}$ & $\Delta \mathrm{CI}$ & DSF & $\Delta \mathrm{DSF}$ & $\mathrm{NI}$ & $\Delta \mathrm{NI}$ & OPI & $\Delta \mathrm{OPI}$ \\
\hline CI & 1.000 & 0.477 & 0.259 & 0.309 & 0.761 & 0.295 & 0.608 & 0.232 \\
\hline$\Delta \mathrm{CI}$ & 0.378 & 1.000 & 0.252 & 0.525 & 0.261 & 0.630 & 0.137 & 0.422 \\
\hline DSF & 0.357 & 0.313 & 1.000 & 0.529 & -0.250 & -0.133 & -0.177 & -0.094 \\
\hline$\Delta \mathrm{DSF}$ & 0.285 & 0.534 & 0.595 & 1.000 & -0.025 & -0.123 & 0.016 & -0.047 \\
\hline NI & 0.823 & 0.203 & -0.237 & -0.066 & 1.000 & 0.394 & 0.771 & 0.303 \\
\hline$\Delta \mathrm{NI}$ & 0.228 & 0.769 & -0.083 & -0.129 & 0.288 & 1.000 & 0.196 & 0.626 \\
\hline OPI & 0.594 & 0.036 & -0.095 & 0.020 & 0.676 & 0.027 & 1.000 & 0.302 \\
\hline$\Delta \mathrm{OPI}$ & 0.122 & 0.446 & -0.021 & -0.007 & 0.140 & 0.529 & 0.176 & 1.000 \\
\hline
\end{tabular}


Tableau 3 (Suite)

Echantillon français $(N=3,586)$

\begin{tabular}{|c|c|c|c|c|c|c|c|c|}
\hline & $\mathrm{CI}$ & $\Delta \mathrm{CI}$ & DSF & $\Delta \mathrm{DSF}$ & NI & $\Delta \mathrm{NI}$ & OPI & $\Delta \mathrm{OPI}$ \\
\hline CI & 1.000 & 0.492 & 0.323 & 0.252 & 0.846 & 0.408 & 0.622 & 0.308 \\
\hline$\Delta \mathrm{CI}$ & 0.445 & 1.000 & 0.271 & 0.525 & 0.355 & 0.726 & 0.249 & 0.508 \\
\hline DSF & 0.397 & 0.319 & 1.000 & 0.529 & -0.063 & -0.017 & -0.098 & -0.021 \\
\hline$\Delta \mathrm{DSF}$ & 0.278 & 0.580 & 0.541 & 1.000 & 0.007 & -0.015 & 0.004 & -0.009 \\
\hline NI & 0.884 & 0.321 & -0.078 & 0.027 & 1.000 & 0.463 & 0.741 & 0.347 \\
\hline$\Delta \mathrm{NI}$ & 0.344 & 0.807 & -0.001 & -0.013 & 0.374 & 1.000 & 0.322 & 0.677 \\
\hline OPI & 0.637 & 0.189 & -0.071 & 0.040 & 0.728 & 0.203 & 1.000 & 0.405 \\
\hline$\Delta \mathrm{OPI}$ & 0.236 & 0.541 & -0.006 & 0.017 & 0.253 & 0.652 & 0.343 & 1.000 \\
\hline
\end{tabular}

Le panel A présente les statistiques univariées pour les variables indépendantes et dépendantes des modèles utilisés dans cette étude. Les statistiques descriptives sont présentées par pays. Les échantillons comprennent toutes les entreprises cotées aux bourses de Londres et Paris dont les données nécessaires au processus de modélisation sont disponibles sur DATASTREAM et WORLDSCOPE pour la période 1992-2004.

Le rendement du titre est le rendement brut annuel du titre de l'entreprise calculé comme le rendement cumulé moyen pour 16,17 et 18 mois, à partir du mois d'ouverture de l'exercice (mois 0). Le return index, la valeur de marché, le résultat net et le résultat opérationnel ont été obtenus à partir de la base DATASTREAM. Le comprehensive income est établi en calculant les variations annuelles de la valeur comptable, i.e. Ordinary Share Capital (DS Item \#301) + Reserves (Item \#304) + Preference Capital (Item \#306), minorées par les titres émis totaux (DS Item \#406) auxquelles sont ajoutés les dividendes versés (DS Item \#434). Toutes les variables comptables sont présentées par action et sont normalisées par le cours de l’action de début de période. Les observations extrêmes comprises dans la tranche des $1 \%$ supérieurs ou inférieurs de la distribution sont éliminées de l'échantillon afin d'éviter des problèmes potentiels de biais par outliers.

Les astérisques indiquent que les moyennes (médianes) du comprehensive income et/ou du résultat opérationnel sont significativement différentes du résultat net quand on utilise un test- $t$ bilatéral (test de Mann-Whitney-Wilcoxon).

Les notations suivantes sont utilisées : ${ }^{*} \mathrm{p}<.1 ;{ }^{* *} \mathrm{p}<.05 ; * * * \mathrm{p}<.01$.

Le panel B présente les coefficients de corrélation obtenus selon la méthode de Pearson et de Spearman pour les variables indépendantes utilisées dans cette étude. Le nombre d’observations pour chaque variable est identique à celui présenté dans le panel A. Les coefficients de corrélation de Pearson et Spearman qui ne sont pas significatifs à un niveau de signification de $10 \%$ sont mis en caractères gras. Comme pour le panel A, toutes les données comptables sont communiquées par action et normalisées par le cours de l'action de début de période.
CI
Comprehensive income
$\Delta \mathrm{CI} \quad$ Variation du Comprehensive income
DSF Dirty surplus flow
$\triangle \mathrm{DSF} \quad$ Variation du Dirty surplus flow
NI Résultat Net (Net Income)
$\Delta \mathrm{NI} \quad$ Variation du Résultat net
OPI Résultat opérationnel (Operating Income)
$\triangle$ OPI Variation du Résultat opérationnel 
Panel B. Résultats de la régression Résultat net / Rendement

Tableau 4. Degré de pertinence (ou value-relevance) du résultat opérationnel, résultat net et comprehensive income

Panel A. Résultats de la régression Résultat opérationnel / Rendement

\begin{tabular}{|l|r|r|r|r|}
\cline { 2 - 5 } \multicolumn{1}{c|}{} & \multicolumn{2}{c|}{ Royaume-Uni } & \multicolumn{2}{c|}{ France } \\
\cline { 2 - 5 }$\beta_{0}$ & Conv. & Rangs & Conv. & Rangs \\
& & & & \\
$\beta_{1}$ & $0.01^{*}$ & $-0.15^{* * *}$ & $0.03^{* * *}$ & $-0.26^{* * *}$ \\
& $(1.90)$ & $(-22.29)$ & $(3.48)$ & $(-18.30)$ \\
$\beta_{2}$ & $0.45^{* * *}$ & $0.27^{* * *}$ & $0.80^{* * *}$ & $0.45^{* * *}$ \\
& $(23.92)$ & $(26.38)$ & $(18.58)$ & $(19.80)$ \\
& $0.20^{* * *}$ & $0.10^{* * *}$ & $0.49^{* * *}$ & $0.28^{* * *}$ \\
$\mathrm{~N}$ & $(7.81)$ & $(9.68)$ & $(8.48)$ & $(12.34)$ \\
& & & & \\
$R^{2}$-ajusté & 7,915 & 7,915 & 4,123 & 4,123 \\
Statistique- $F$ & $8.32 \%$ & $11.55 \%$ & $11.32 \%$ & $16.59 \%$ \\
& $359.98^{* * *}$ & $517.81^{* * *}$ & $264.07 * * *$ & $411.07 * * *$ \\
\hline
\end{tabular}

\begin{tabular}{|l|r:r|r|r|}
\cline { 2 - 5 } \multicolumn{1}{c|}{} & \multicolumn{2}{c|}{ Royaume-Uni } & \multicolumn{2}{c|}{ France } \\
\cline { 2 - 5 }$\beta_{0}$ & Conv. & Rangs & Conv. & \multicolumn{1}{c|}{ Rangs } \\
\hline$\beta_{1}$ & $0.03^{* * *}$ & $-0.13^{* * *}$ & $0.05^{* * *}$ & $-0.31^{* * *}$ \\
& $(10.14)$ & $(-18.54)$ & $(6.54)$ & $(-22.05)$ \\
$\beta_{2}$ & $0.27^{* * *}$ & $0.26^{* * *}$ & $1.13^{* * *}$ & $0.55^{* * *}$ \\
& $(17.37)$ & $(23.91)$ & $(19.68)$ & $(23.01)$ \\
& & & & \\
$\mathrm{N}$ & $\left(2.03^{* *}\right.$ & $0.06^{* * *}$ & $0.35 * * *$ & $0.27 * * *$ \\
& & $(5.08)$ & $(6.54)$ & $(11.28)$ \\
$R^{2}$-ajusté & 6,983 & & & \\
Statistique- $F$ & $4.88 \%$ & $10.42 \%$ & $13.60 \%$ & $23.68 \%$ \\
& $180.22^{* * *}$ & $407.04^{* * *}$ & $281.59 * * *$ & $553.90^{* * *}$ \\
\hline
\end{tabular}

Panel C. Résultats de la régression Comprehensive Income / Rendement

\begin{tabular}{|c|c|c|c|c|}
\hline & \multicolumn{2}{|c|}{ Royaume-Uni } & \multicolumn{2}{|c|}{ France } \\
\hline & Conv. : & Rangs & Conv. & Rangs \\
\hline$\beta_{0}$ & $\begin{array}{r:}0.03^{* * * *} \\
(9.92)\end{array}$ & $\begin{array}{r}-0.09 * * * \\
(-12.40)\end{array}$ & $\begin{array}{r}0.06 * * * \\
(8.36)\end{array}$ & $\begin{array}{r}-0.23 * * * \\
(-15.89)\end{array}$ \\
\hline$\beta_{1}$ & $\begin{array}{r:}0.20^{* * *} \\
(14.19)\end{array}$ & $\begin{array}{c}0.23^{* * *} \\
(19.30)\end{array}$ & $\begin{array}{r}0.70^{* * * *} \\
(14.66)\end{array}$ & $\begin{array}{r}0.49 * * * \\
(19.43)\end{array}$ \\
\hline$\beta_{2}$ & $\begin{array}{r:}0.03^{* * * *} \\
(3.09)\end{array}$ & $\begin{array}{r}0.01 \\
(0.58)\end{array}$ & $\begin{array}{r}0.14 * * * \\
(3.76)\end{array}$ & $\begin{array}{r}0.15^{* * *} \\
(6.08)\end{array}$ \\
\hline $\mathrm{N}$ & 7,186 & 7,186 & 3,865 & 3,865 \\
\hline$R^{2}$-ajusté & $3.57 \%$ & $6.38 \%$ & $7.64 \%$ & $14.92 \%$ \\
\hline Statistique- $F$ & $134.02^{* * *}$ & $209.90 * * *$ & $160.84 * * *$ & $339.85 * * *$ \\
\hline
\end{tabular}


Tableau 4 (Suite)

Panel D. Résultats de la régression des Dirty surplus flows / Rendement

\begin{tabular}{|c|c|c|c|c|}
\hline & \multicolumn{2}{|c|}{ Royaume-Uni } & \multicolumn{2}{|c|}{ France } \\
\hline & Conv. & Rangs & Conv. & Rangs \\
\hline$\beta_{0}$ & $\begin{array}{r}0.03^{* * *} \\
(10.12)\end{array}$ & $\begin{array}{r}-0.13^{* * *} \\
(-13.56)\end{array}$ & $\begin{array}{r}0.05^{* * *} \\
(6.71)\end{array}$ & $\begin{array}{r}-0.33^{* * *} \\
(-17.44)\end{array}$ \\
\hline$\beta_{1}$ & $\begin{array}{r}0.27 * * * \\
(16.60)\end{array}$ & $\begin{array}{r}0.25^{* * *} \\
(22.37)\end{array}$ & $\begin{array}{r}1.11^{* * *} \\
(19.20)\end{array}$ & $\begin{array}{r}0.54 * * * \\
(22.69)\end{array}$ \\
\hline$\beta_{2}$ & $\begin{array}{r}0.04 * * * \\
(2.94)\end{array}$ & $\begin{array}{r}0.06 * * * \\
(5.47)\end{array}$ & $\begin{array}{r}0.35 * * * \\
(6.61)\end{array}$ & $\begin{array}{r}0.27 * * * \\
(11.49)\end{array}$ \\
\hline$\beta_{3}$ & $\begin{array}{r}-0.04 \\
(-1.21)\end{array}$ & $\begin{array}{r}-0.03 * * * \\
(-2.76)\end{array}$ & $\begin{array}{r}-0.26^{* *} \\
(-2.02)\end{array}$ & $\begin{array}{r}-0.08 * * * \\
(-3.10)\end{array}$ \\
\hline$\beta_{4}$ & $\begin{array}{r}0.09 * * * \\
(3.91)\end{array}$ & $\begin{array}{r}0.05 * * * \\
(4.08)\end{array}$ & $\begin{array}{r}0.18^{* *} \\
(2.13)\end{array}$ & $\begin{array}{r}0.11 * * * \\
(4.53)\end{array}$ \\
\hline $\begin{array}{l}\mathrm{N} \\
R^{2} \text {-ajusté } \\
\text { Statistique-F }\end{array}$ & $\begin{array}{r}6,983 \\
5.09 \% \\
94.70 * * *\end{array}$ & $\begin{array}{r}6,983 \\
10.61 \% \\
208.26^{* * *}\end{array}$ & $\begin{array}{r}3,564 \\
13.69 \% \\
142.34 * * *\end{array}$ & $\begin{array}{r}3,564 \\
24.09 \% \\
283.77 * * *\end{array}$ \\
\hline$\beta_{3}+\beta_{4}$ & $\begin{array}{r}0.04 * * * \\
(3.32)\end{array}$ & $\begin{array}{r}0.02^{* *} \\
(2.15)\end{array}$ & $\begin{array}{r}0.02 \\
(0.39)\end{array}$ & $\begin{array}{r}0.03 \\
(1.25)\end{array}$ \\
\hline
\end{tabular}

Le tableau 4 décrit les résultats empiriques de la régression MCO suivante : $R E T_{t}=\beta_{0}+\beta_{1} \frac{R C_{t}}{P_{t-1}}+\beta_{2} \frac{\Delta R C_{t}}{P_{t-1}}+u_{t} \quad$ avec $R E T_{t}$ le rendement brut des titres de l'entreprise; $R C_{t}$, la mesure du résultat comptable par titre, i.e. résultat opérationnel, résultat net et comprehensive income ; et $\Delta R C_{t}$, la variation du résultat comptable par titre pour chaque période financière $t$. $R E T_{t}$ est calculé ici comme le rendement cumulé moyen des titres pour 16, 17 et 18 mois, à partir du mois d'ouverture de l'exercice (mois 0 ). Toutes les variables à l'exception de $R E T_{t}$ sont normalisées par les cours des titres à la fin de l'exercice précédent $P_{t-1}$. Le panel A présente les coefficients et les statistiques-t de la régression du résultat opérationnel, selon la méthode conventionnelle des MCO et la méthode des rangs pour chaque pays. A l'instar d’Abarbanell \& Bushee (1988), nous avons calculé les rangs (par décile) pour chaque variable indépendante utilisée dans l'équation (6a) en classant les valeurs des variables dans dix portefeuilles (avec les rangs 0 à 9). Les déciles obtenus sont ensuite normalisés par neuf afin que la variable indépendante obtenue soit comprise dans un intervalle unitaire positif et normalisé. Le panel $B$ et $C$ présente respectivement les résultats de la régression pour le résultat net et le comprehensive income. Le panel $\mathrm{D}$ présente les résultats de la régression suivante :

$R E T_{t}=\beta_{0}+\beta_{1} \frac{N I_{t}}{P_{t-1}}+\beta_{2} \frac{\Delta N I_{t}}{P_{t-1}}+\beta_{3} \frac{D S F_{t}}{P_{t-1}}+\beta_{4} \frac{\Delta D S F_{t}}{P_{t-1}}+u_{t}$

avec $R E T_{t}$ le rendement brut des titres de l'entreprise ; $N I_{t}$, le résultat net par titre ; et $\Delta N I_{t}$, la variation du résultat net par titre pour chaque période financière $t ; D S F_{t}$, le flux de dirty surplus ; et $\triangle D S F_{t}$, la variation du flux de dirty surplus.

Les notations suivantes sont utilisées dans tous les panels :

${ }^{*} \mathrm{p}<.1 ;{ }^{* *} \mathrm{p}<.05 ; * * * \mathrm{p}<.01$. 


\section{Tableau 5. Comparaison des $\boldsymbol{R}^{2}$ de l'échantillon britannique avec ceux des échantillons européens}

Le tableau 5 présente la statistique $R^{2}$-ajusté et la statistique-z de Vuong (1989) utilisées dans la discussion des hypothèses 1 et 2 de notre cadre de recherche. Le panel A présente la statistique $R^{2}$-ajusté pour chacun des quatre modèles de résultat estimés à partir des régressions MCO selon la méthode conventionnelle et la méthode des rangs. Les résultats sont présentés par pays. Les quatre dernières colonnes montrent la statistique $R^{2}$-ajusté moyenne (médiane) pour chaque mesure du résultat (earnings components) utilisée dans cette étude. Le panel B présente la statistique-z de Vuong (1989) utilisée afin de tester la différence de signification entre nos différents modèles non-imbriqués sur les éléments de la performance. Ces modèles sont représentés par leurs variables dépendantes, i.e. respectivement $C I, N I+D S F$, NI et $O P I N$. Les notations suivantes sont utilisées : ${ }^{*} \mathrm{p}<.1$; $* * \mathrm{p}<.05 ; * * * \mathrm{p}<.01$.

Panel A. $R^{2}$-ajusté (en \%) pour les modèles de composants de résultat

\begin{tabular}{|c|c|c|c|c|}
\hline $\begin{array}{l}\text { Variable(s) } \\
\text { Indépendante(s) }\end{array}$ & $\begin{array}{l}\text { Méthode de } \\
\text { regression }\end{array}$ & $\begin{array}{l}\text { Royaume-Uni } \\
\text { (RU) }\end{array}$ & $\begin{array}{c}\text { France } \\
\text { (FR) }\end{array}$ & $\begin{array}{c}\text { Différence: } \\
\text { FR-RU }\end{array}$ \\
\hline Model (6c): & Conventionnelle & 3.57 & 7.64 & +4.07 \\
\hline Comprehensive Income & Rangs & 6.38 & 14.92 & +8.54 \\
\hline Model (6d): Résultat net & Conventionnelle & 5.09 & 13.69 & +8.60 \\
\hline et Dirty Surplus Flow & Rangs & 10.61 & 24.09 & +13.48 \\
\hline Model (6b): & Conventionnelle & 4.88 & 13.60 & +8.72 \\
\hline Résultat net & Rangs & 10.42 & 23.68 & +13.26 \\
\hline Model (6a): & Conventionnelle & 8.32 & 11.32 & +3.00 \\
\hline Résultat opérationnel & Rangs & 11.55 & 16.59 & +5.04 \\
\hline
\end{tabular}

Panel B: Statistique-z deVuong (1989) pour des modèles non-imbriqués des composants de la performance

\begin{tabular}{|c|c|c|c|c|c|}
\hline \multirow{2}{*}{\multicolumn{2}{|c|}{ Hypothèse nulle }} & \multicolumn{2}{|c|}{ Royaume-Uni } & \multicolumn{2}{|c|}{ France } \\
\hline & & Conv. & Rangs & Conv. & Rangs \\
\hline \multirow[t]{4}{*}{$\mathrm{NI}>\mathrm{CI}$} & Likelihood ratio & 48.00 & 144.88 & 76.70 & 151.31 \\
\hline & Variance $\omega^{2}$ & 0.03 & 0.05 & 0.06 & 0.11 \\
\hline & Statistique $z$ & $3.30 * * *$ & $7.61 * * *$ & $5.43 * * *$ & $7.52 * * *$ \\
\hline & $\mathrm{N}$ & 6,841 & 6,841 & 3,523 & 3,523 \\
\hline \multirow[t]{4}{*}{ OPI $>$ CI } & Likelihood ratio & 156.97 & 192.54 & 64.56 & 38.46 \\
\hline & Variance $\omega^{2}$ & 0.10 & 0.10 & 0.12 & 0.18 \\
\hline & Statistique $z$ & $6.05^{* * *}$ & $7.51 * * *$ & $3.10 * * *$ & $1.54^{*}$ \\
\hline & $\mathrm{N}$ & 6,841 & 6,841 & 3,523 & 3,523 \\
\hline \multirow[t]{4}{*}{ NI $>$ OPI } & Likelihood ratio & -108.97 & -47.67 & 12.14 & 112.86 \\
\hline & Variance $\omega^{2}$ & 0.09 & 0.06 & 0.11 & 0.15 \\
\hline & Statistique $z$ & $-4.30 * * *$ & $-2.31 * *$ & 0.63 & $4.96 * * *$ \\
\hline & $\mathrm{N}$ & 6,841 & 6,841 & 3,523 & 3,523 \\
\hline
\end{tabular}




\section{NOTES}

${ }^{1}$ Cf. Escaffre L. et Ramond O. (2005) : Les enjeux de l'introduction du «Comprehensive Income » dans les normes IFRS : Vers une représentation de la performance globale des entreprises, Option Finance, 6 juin 2005.

2 “ [...] the ideal set of accounting rules is one that makes the price-earnings ratio as constant as possible" (Black, Financial Analysts Journal, novembre 1980: 19).

${ }^{3}$ Projet publié en mars 2006 qui s’inscrit dans la phase I du projet Performance reporting.

${ }^{4}$ Règlement n99-02 du 29 avril 1999 (modifié) relatif aux comptes consolidés des sociétés commerciales et entreprises publiques.

${ }^{5}$ En effet, FAS 130 laisse ouvert le choix d’inclure les éléments du CI dans le tableau de variation des capitaux propres ou dans un document spécifique.

${ }^{6}$ En vue de faciliter la lecture des équations, l’indice de l’entreprise a été supprimé.

${ }^{7}$ Dans cette étude, nous calculons le rendement des titres au début de la période financière à 4, 5, 6 mois après la fin de l'année pour chaque entreprise. Nous prenons ensuite la moyenne des trois valeurs pour chaque observation. Trois horizons temporels sont ainsi utilisés pour le calcul de notre variable dépendante, car nous soupçonnons certaines sociétés de divulguer les informations relatives à leurs résultats plus tardivement que d'autres. Si cette hypothèse était avérée, un décalage d’impact des résultats sur le rendement dans notre modèle pourrait être observée et biaiser par nos estimateurs statistiques. D’autres variables proxies pour le rendement des titres ont été également utilisés pour réaliser des tests de sensibilité.

${ }^{8}$ La période d'analyse est raccourcie d'une année (i.e. 1993-2004) lors de la construction de la variable comprehensive income basée sur une variation de capitaux propres (nécessitant deux périodes d'observation).

${ }^{9}$ Néanmoins, l'année 2005 a fait objet de tests de sensibilité dont les résultats ne sont pas différents de ceux observés sur la période pré-IFRS.

${ }^{10}$ INDC6 est la classification industrielle la plus détaillée de DATASTREAM. Cette analyse industrielle est basée sur la nouvelle classification FTSE/DJ (similaire au schéma de classification de $4{ }^{\text {ème }}$ degré US SIC 4) et comprend 83 sous-sections sectorielles.

${ }^{11}$ Par ailleurs, il est important de noter que la phase B du projet «Performance Reporting » de l'IASB « [...] se concentre d'abord sur les sociétés non financières [...et] les spécificités de la communication financière des sociétés financières seront étudiées ultérieurement " (IASB, Financial Statement for Observers: Financial Statement Presentation, page 3, paragraph 9, 26 ${ }^{\text {th }}$ of July 2006). De même, la norme UK FRS 3 ne s'applique qu'aux sociétés non financières. Les sociétés financières sont donc exclues de cette étude dans un souci de cohérence.

12 Cette hypothèse a été relaxée dans les tests de sensibilité et n’a pas permis de constater une différence significative sur les résultats de nos modèles.

${ }^{13}$ En vue d’alléger notre discussion, nous utiliserons le nom des pays pour dénommer nos échantillons.

${ }^{14}$ Une corrélation négative similaire est communiquée bien que non discutée dans l'étude américaine de Cheng et al. (1993). 